\title{
Review
}

\section{Return to the RNAi world: rethinking gene expression and evolution}

\author{
Nobel Lecture, December 8, 2006 \\ by CC Mello
}

\begin{abstract}
Thanks to the Nobel Foundation for permission to publish this Lecture (Copyright ${ }^{\complement}$ The Nobel Foundation 2006). Here we report the transcript of the lecture delivered by Professor Craig C Mello at the Nobel Prize ceremony. Professor Mello vividly describes the years of research that led to the discovery of RNA interference and the molecular mechanisms that regulate this fundamental cellular process. The turning point of discoveries and the role played by all his colleagues and collaborators are described, making this a wonderful report of the adventure of research. The lecture explains in simple language the importance of this discovery that has added a great level of complexity to the way cells regulate protein levels; moreover, it points out the beauty and importance of Caenorhabditis elegans as a model organism and how the use of this model has greatly contributed to the advance of science. Finally, Professor Mello leaves us with a number of questions that his research has raised and that will require years of future research to be answered.
\end{abstract}

Cell Death and Differentiation (2007) 14, 2013-2020; doi:10.1038/sj.cdd.4402252

Its wonderful to be here today, I would like to start with the most important part, by saying thank you. First of all, I want to thank Andy Fire for being such a tremendous colleague, friend and collaborator going back over the years. Without Andy I definitely would not be here today. I need to thank the University of Massachusetts for providing for my laboratory, for believing in me and for giving me not only a place and money, but great colleagues with whom to pursue my research. Without UMass and the great environment provided for me there, I probably would not be here today. And, of course my family; I am not going to spend time now thanking them individually, but they know how important they are.

I am going to talk today about Caenorhabditis elegans and the role of RNAi in $C$. elegans development. This animal is aptly named for its elegant simplicity. It is only $1 \mathrm{~mm}$ in length and yet capable of producing 300 progeny in 3 days by selffertilization. One of the most beautiful things about $C$. elegans, immediately apparent upon viewing it in the microscope, is its transparency. Sydney Brenner recognized the importance of this attribute when deciding what organism to work on. As animals go, $C$. elegans is relatively simple, having only about a thousand cells in the adult organism. Indeed, the origin and fate of every cell, both in the embryo and adult, has been determined - an amazing accomplishment. At any stage of development, you can look at a cell and know where that cell came from, tracing its origin back in time to the first division of the embryo.
Its a beautiful system. In fact, the researchers who work on C. elegans have their own lineage. Almost all of us can trace ourselves back to Sydney Brenner, who pioneered the modern genetic analysis of this organism. My particular ancestors, if you will, in the lineage of researchers are shown in Figure 1. I owe a tremendous amount of thanks to Dan Stinchcomb, for teaching me molecular biology and really being a fantastic mentor during my initial years in graduate school; Victor Ambros who, along with Dan, provided a wonderful joint laboratory at Harvard University where I did graduate work; and then Jim Priess, who taught me genetics, and was a tremendous mentor and a great friend out in Seattle where I conducted my postdoctoral research at the Fred Hutchinson Cancer Research Center. I owe a tremendous amount to these individuals. I will show you more pictures of people I will need to thank as I go along.

I want to get down to the theme of my talk today, which really is, in part, about how we continually underestimate the complexity of life. Its the correction of these underestimations that is quite often what this prize is really recognizing. As science progresses, our knowledge expands, we think we understand, and too often we become overconfident. The fact is, I think we almost always underestimate the complexity of life and of nature. Today has been a true celebration of that beauty and complexity. I attended the Physics talks and the Chemistry talks and it was just spectacular to contemplate. An embryonic universe 13.7 billion years old, originally on the

\footnotetext{
${ }^{1}$ Howard Hughes Medical Institute and Program in Molecular Medicine, University of Massachusetts Medical School, Worcester, MA, USA Correspondence: G Melino, Cell Death and Differentiation, Rome Editorial Office, D26, University Rome Tor Vergata, via Montpellier 1, 00133-Rome, Italy. E-mail: cell.death.differ@uniroma2.it
}

Keywords: RNAi; siRNA; C. elegans; argonaute family; rde-1; transcription; cancer 


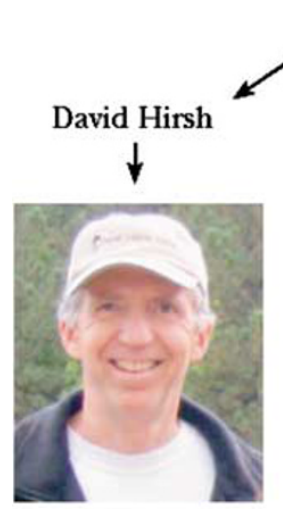

Dan Stinchcomb
Sidney Brenner

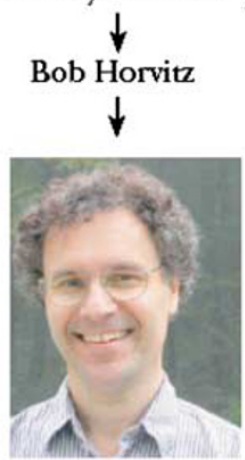

Victor Ambros

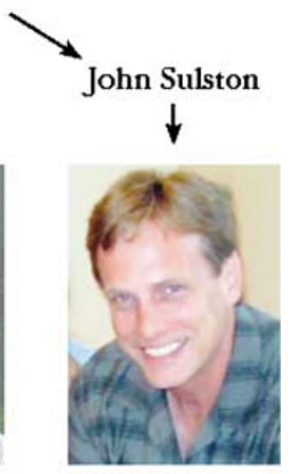

Jim Priess

Figure 1

scale of inches across, expanding in seconds through a mysterious process of inflation to occupy the nearly infinite dimensions of space. And to explore the workings of a polymerase at the atomic level, whose origins derive from a common ancestor of all life on Earth some 3.5 billion years ago.

These stories are so beautiful and stunning in their complexity. For every answer they provide they raise a thousand new questions. And so one thing l'd like to accomplish in my talk, is to raise questions that I cannot answer, and to talk about the unknown some more. Andy has done such a wonderful job of introducing the subject, giving me the luxury of spending some time talking about potential implications and some of the things we do not know, but Sydney Brenner, David Hirsh, Bob Horvitz, John Sulston, Dan Stinchcomb, Victor Ambros, Jim Priess would love to understand in the future. It is the unknown that inspires us and sparks our curiosity, and so I would like to try to focus more on telling you about what we do not know and on speculating on what is possible.

If one looks carefully, the complexity of living things becomes strikingly clear. Consider for example the natural environment of $C$. elegans. Figure 2 is an electron micrograph taken by George Barron, who works on nematophagous fungi. The unfortunate worm shown here has become ensnared in a trap set by a fungus that preys on nematodes. It really is a jungle out there for these poor little animals; they struggle to survive, just like the rest of us. The soil is filled with hundreds of different species of these fungi that prey on worms as they are swimming around in the soil. These fungi can sense the motion or contact of a worm and, after the worm has entered its lariat, the fungus inflates it to constrict the snare around the animal, trapping it. The fungus can then send hyphae into the worm to digest it. So, imagine that these poor elegant little animals are actually struggling to survive out there. Nature is filled with complexity that we do not appreciate. This is so tiny, that you would walk over millions, if not billions, of these little creatures in the soil every day on your way to work, never realizing the things that are happening there.

One of the great triumphs of biology was the discovery of the structure of DNA. The structure of DNA was first determined by Watson and Crick, who showed how two strands composed of four basic building blocks form polymers

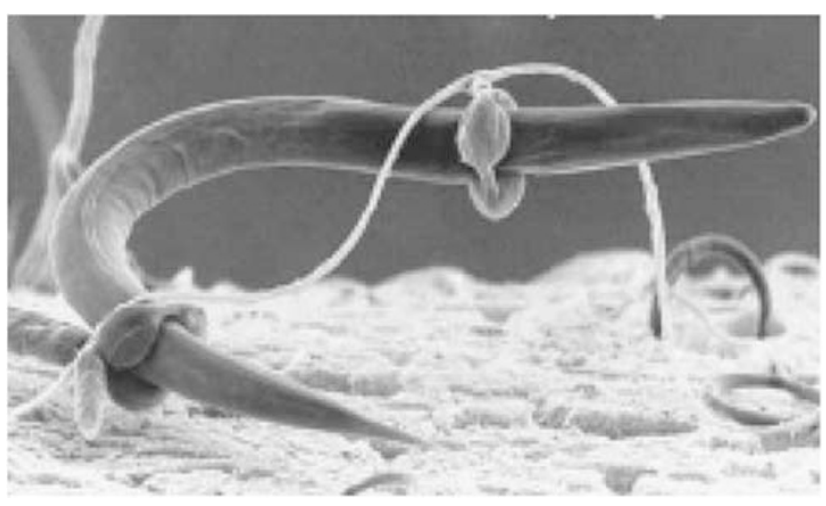

Figure 2 C. elegans captured by fungi. Photo by George Barron.

that intertwine in a beautiful helical staircase structure. This structure explains so much, really, about the basic biology of living things. It explains the segregation patterns, first described by Gregor Mendel, for certain genetic traits of pea plants. The structure alone, as Watson and Crick noted, suggests how the genetic material can be replicated. They stated in their famously brief paper in Nature 1 that, 'It has not escaped our notice that the specific pairing we have postulated immediately suggests a possible copying mechanism for the genetic material.' The DNA strands are wrapped around each other and each can template the production of a perfect copy of the strand to which it is bound simply by unwinding and allowing the polymerase to copy it. In Roger Kornberg's talk, we heard about an RNA polymerase that can transcribe the DNA to produce RNA copies of the genetic information. These copies provide templates for the polymerization of the proteins through another elaborate and really beautiful process, called translation, that I certainly do not have time to describe today. I would hope that, if you are interested in these basic workings of the cell, which certainly I think everyone should be interested in, you should look at the literature and do some searching on the Internet to learn more about this process - it is truly amazing.

But, one of the problems with a discovery like this one, of DNA, is that we tend to become overconfident in the explanatory power of the discovery. Does the DNA sequence information control all of the events in the cell? Cells are constantly responding to their environment and to surrounding cells, and these external influences can alter the cell in heritable ways that do not require changes in the primary sequence information in the DNA. Consider the early $C$. elegans embryo. During these early divisions, maternal mRNA and protein products that are stored in the egg direct numerous cell-cell signaling and differentiation events that give rise to the multicellular organism. These are exemplified by the distribution of the PIE-1 protein. PIE-1 tracks with, and is essential for, germline specification. As shown in this image from a movie, PIE-1 - in this case tagged with a glowing jellyfish protein - becomes localized after each division to the germline cell. In this two-cell embryo PIE-1 protein is localized exclusively to the posterior cell where it is concentrated in the nucleus. This occurs through a fundamental developmental 
process called asymmetric (unequal) cell division. As a result of this process, the two daughter cells differ with respect to their content of maternally provided products, like PIE-1. These products, in turn, can direct the subsequent development of these cells such that, once differentiated in this way, these cells remain committed to their specific tasks in the animal through numerous rounds of cell division. These remarkably stable differentiation events can be maintained for the entire life of an organism without any underlying changes in the DNA sequence. The germline cells, which in $C$. elegans inherit PIE-1 protein, are the only cells that retain the potential to launch the developmental program again in the next generation.

How do developing cells, all with the same DNA content, lock in different programs of gene expression that are stable through so many rounds of cell division? One possibility, as I will discuss below, is that mechanisms related to those that mediate RNA interference have a role in this process. It has been suggested that the origin of life on Earth may have begun with self-replicating nucleic acid polymers that were more similar chemically to RNA than to DNA, a classic hypothesis referred to as the 'RNA World' hypothesis. Hence, the provocative definition 'Return to the RNA World,' a world in which RNA molecules may have carried, and may still carry, genetic information. The direct ancestors through cell division of the $C$. elegans germ cells were primordial germ cells in the common metazoan (probably worm-like) ancestor of worms and humans, and going even farther back are direct descendants of the hypothetical self-replicating RNA molecules that gave rise to all life on Earth some 3.5 billion years ago. We heard earlier today, in the physics lectures, that the temperature of the cosmic-background radiation is consistent with an age for the universe of 13.7 billion years. Thus, life on earth is about a quarter of the age of the universe. Living things and these mechanisms that we are talking about today are incredibly ancient. RNAi itself is at least one billion years old. Biological mechanisms are far more constant than the positions of continents on our planet. That fact and the implicit concept of deep time are among the most profound discoveries of science.

Considering the possible origins of life in a world where information was stored in RNA polymers, and considering the remarkable sophistication of living things and the constancy of the basic and fundamental principles underlying mechanisms of biology, and finally, considering what we now know about RNA and RNA interference, it is perhaps a good time to reconsider the idea that genetic information is stored primarily in the nucleotide sequence of our DNA. In thinking about this, it is interesting to consider what previous scientists thought about the mechanism of inheritance before DNA and RNA were discovered. For example, in the late 1800s August Weismann, a famous naturalist and early thinker on mechanisms of inheritance, coined the term 'biophore' to describe the hereditary agent. ${ }^{2}$ Ernst Mayr, in describing Weismann's work in his book The Growth of Biological Thought, characterizes Weismann's ideas as flawed. Weismann said that '(1) there is a special particle, the biophore, for each trait; (2) that these particles can grow and multiply independent of cell division; (3) that both the nucleus and cytoplasm consist of these biophores; (4) that a given biophore may be represented by many replicas in a single nucleus, including the germ cell; and (5) that during cell division the daughter cells may receive different kinds and numbers of biophores through unequal cell division'. ${ }^{3}$ Mayr concludes that 'As we now know' (thanks to Mendel), 'postulates (2) and (5) are wrong and are responsible for the fact that Weismann was not able to arrive at a correct theory of inheritance.'

Well, are they really wrong? If you try to apply Weismann's concepts to all genes or genetic traits they are clearly not adequate to explain inheritance. For example, Weismann's biophores could not explain the striking segregation patterns first observed by Mendel for the genetic traits of pea plants. Thus, yes, it would be wrong to apply Weismann's theory to define all genetic inheritance. But let's consider applying Weismann's theory to some traits, and then replace the term 'biophores' with the term 'siRNAs.' Andy introduced siRNAs as these small interfering RNAs, as we call them; the little chunks of RNA that go on and silence genes. If we put 'siRNAs' into each place where 'biophores' appears in Weismann's theory, we then have a very different situation: (1) there is a particle, containing siRNAs, for some traits; (2) these siRNAs can grow and multiply independent of cell division; (3) both the nucleus and the cytoplasm can contain the siRNAs; (4) a given siRNA may be represented by many replicas; and (5) that during cell division the daughter cells may receive different kinds and numbers of siRNAs through unequal cell division. And with these changes, and in light of what we now know about RNAi (as will be discussed more below), it becomes clear that these postulates are not necessarily wrong. Weismann had some very good ideas and we should not discard them out of hand. RNA may play a role in inheritance and evolution. I will talk about a mechanism for RNA-directed inheritance toward the end of my talk. Furthermore, I will suggest how natural variation in silencing levels could underlie heritable phenotypic variation upon which evolution could act.

To help introduce RNAi, I am going to describe some movies that try to capture the essence of the RNAi process. Andy and I take hours to explain RNAi, but that will not do for today's television audience. It is a major problem for television programmers, as you may know. People watch with the remote control handy at all times, so you have to get your point across very quickly, before your viewer loses interest and clicks to another channel. Consequently, in the television industry, they are very good at making models and graphics that can show complex mechanisms like RNAi in just a few seconds.

So, here is what CBS Evening News came up with to try to explain RNAi. For the average viewer at prime time the attention span is about fifteen seconds. So, here's what CBS Evening News came up with. In the movie, the doublestranded RNA flies onto the scene then opens at one end and begins to open and close as though it is chewing. Defective genes, shaped like colored cheese puffs, then begin to fly into the mouth of the RNA from the left. The RNA is literally eating the DNA for lunch. Now, Andy and I knew that RNA interference was something incredible when we started working on it, but we really did not have any idea that it was this incredible. Of course there are a few details that are glossed over in this explanation. 
Public broadcasting has the luxury of an audience that tends to have a bit more patience, and they came up with a 15 min segment and another strategy, 'the cop', to explain RNAi. They describe a little policeman who looks out for viruses and other misbehavior in the cell. When he sees double-stranded RNA he realizes something is not right. $\mathrm{He}$ then goes on to use 'enzymatic kung fu' to destroy not only the dsRNA with that sequence, but all RNAs with that sequence that he encounters in the cell.

I like both of these movies because they illustrate a really important concept; that is, that RNAi is an active process, that there is an organismal response to the dsRNA. ${ }^{4}$ We realized this at an early stage, because, first of all, as Andy mentioned, the silencing was heritable. RNA injected into an animal resulted in silencing that was transmitted to progeny and even transmitted through crosses for multiple generations via the egg or the sperm. So, the interference mechanism can be initiated in one generation and then transmitted in the germ line. And, interestingly, RNAi is also systemic; RNA injected anywhere in the body, or even delivered by ingestion, can get into all the tissues, including the germline. So RNAi involves a transport mechanism, meaning it can be transferred from cell to cell in the body.

The inheritance properties and systemic nature of RNAi, along with its remarkable potency in $C$. elegans, all pointed toward an active organismal response to the double-stranded RNA. What we wanted to do immediately, upon realizing that there was an active response in the organism, was to find the genes in the animal that encode that response. Therefore, we set out to use the powerful genetics of $C$. elegans to look for mutant strains defective in RNAi. We imagined that these mutants would define genes required for the recognition of the foreign double-stranded RNA, genes required for the transport of the silencing signal from cell to cell, genes required for the amplification of silencing, and genes required for the silencing apparatus itself.

Hiroaki Tabara, was the first person to do RNAi genetics in the world. He was a courageous postdoc who came to my lab to study development, but was willing to tackle something as unusual and as odd as RNAi. The screen that he did was very simple. Basically, he mutagenized animals, let them grow for two generations until mutations that had been induced would become homozygous and then, using a trick first developed by Lisa Timmons in Andy's lab, ${ }^{5}$ he fed the worms $E$. coli expressing double-stranded RNA targeting an essential worm gene. According to this strategy, if the animals have an intact RNAi response, then RNAi would knock out the activity of the essential gene, causing lethality. Now, if by chance a mutant exists in the population that lacks an RNAi response, then RNAi would not occur, and the corresponding animal and its progeny would be viable. Hiroaki used this very powerful genetic selection to identify mutants defective in RNAi, and his screen worked very, very well. Hiroaki was able to identify numerous mutants. Some of these lacked the RNAi response and had no other obvious phenotypes, like rde-1 and rde-4. However, some of his mutants had additional defects, including a very striking phenotype in which the transposons, which are self-replicating DNA elements present in the genomes of all organisms, became hyperactive, causing mutations by jumping from place to place in the genome. In addition, these same mutants had a reduced tendency to silence transgenes in the germ line (transgenes are genes that are experimentally introduced into the organism). In normal worms, transgenes have the vexing property of becoming silent after introduction into the animal experimentally. The same mutants with activated transposons also exhibited activation of transgenes in the germ line.

These observations suggested that the normal physiological function of RNAi might be to defend cells against the potentially damaging effects of transposons and other foreign genetic elements (perhaps including viruses). However, there was a big problem with this relatively simple model. The rde- 1 and rde-4 mutants, as I indicated earlier, had no other phenotypes. They were strongly deficient in RNAi in response to double-stranded RNA, but the transposon silencing and the transgene silencing mechanisms were still functioning in these mutant strains. These observations indicated to us, even at that very early stage of our analysis, the existence of some additional, very interesting complexity. The rest of the science that I will discuss below really relates to our further investigation of this complexity, and to how these investigations led to the realization that related silencing pathways with distinct triggering mechanisms are at work in $C$. elegans. Keeping in mind that the Nobel committee was careful to recognize, very specifically, the initiation of gene silencing by double-stranded RNA, I hope that we can look forward to future recognition of silencing that is triggered in other ways. For example, silencing driven from endogenous dsRNAencoding genes, microRNAs, or silencing triggered by the introduction of transgenes.

Hiroaki cloned the rde-1 gene and showed that it encodes a highly conserved protein that we now refer to as an Argonaute protein. ${ }^{6}$ RDE-1 was an interesting protein for a couple of reasons. It had highly conserved domains found in related genes in organisms as diverse as plants and humans, and yet nothing was known about the enzymatic activities or the biological functions of these domains. This was a very exciting time in the laboratory. We at last had a gene that we knew was involved in the mechanism. Furthermore, previous work on one gene closely related to RDE-1 from Drosophila had linked this gene family to an epigenetic silencing pathway in the fruit fly, ${ }^{7,8}$ and work in plants had linked a member of the family to the control of development. ${ }^{9}$ Very shortly after our paper was published, Carlo Cogoni and Giuseppe Macino ${ }^{10}$ published a very nice paper implicating an RDE-1 family member in silencing triggered by the introduction of a transgene in the fungus Neurospora. So from these findings in other organisms, and from Hiroaki's genetics, we hypothesized that there may be other types of triggers that initiate related silencing pathways either through natural developmental mechanisms or in response to transposons and transgenes.

A very exciting possibility occurred to us after we cloned rde-1. To explain this possibility, I first have to describe some fundamental facts about genes and how the amazingly successful genome-sequencing projects around the world have impacted biological research. Genes are composed of long sequences of nucleotides that specify the protein-coding potential and/or other functions of their gene products. The relationships between genes can be inferred by looking at the nucleotide sequence of the gene. For example, by using the 
nucleotide sequence to infer the protein-coding potential of all the known genes related to rde-1, it was possible to build what is called a phylogenetic tree (Figure 3 ), in which the most similar members of the gene family (often referred to as homologs) are closest to each other on the tree. Interestingly, it turns out that $r d e-1$ is a member of a large gene family, with 26 related genes in $C$. elegans. Similarly, there are multiple Argonaute genes in almost every organism. The organisms from which each gene in the tree is derived are indicated by a prefix as follows: $C$. elegans $(\mathrm{Ce})$, humans $(\mathrm{Hs})$, the plant $A$. thaliana (At), fruit fly (Dm), and fission yeast (Sp 250). The genes named in black represent the most highly conserved branch of the family, with members in plants, animals, and fungi. The green branch, often referred to as the Piwi family after its founding member, has family members in all animals (but not in plants or fungi). Finally, the red branch of the tree represents a $C$. elegans-specific subfamily of genes that are equally divergent from both the black and green families. This remarkable diversity of Argonaute genes raised the exciting possibility that different members of the family have become specialized in each organism to perform distinct functions. For example, RDE-1 according to our genetic studies is required for gene silencing in response to foreign dsRNA. Perhaps, other members of the $C$. elegans Argonaute gene family mediate transposon and transgene silencing. Still others may function in developmental pathways related to RNAi. An outstanding graduate student, Alla Grishok, took on the task of trying to test these ideas.

To do this, Alla set out to inactivate members of the C. elegans Argonaute gene family. The first genes that she knocked out encoded two closely related members of the most highly conserved group of Argonautes, named ALG-1 and ALG-2 (see the black branch of the tree, Figure 3).

When Alla knocked out these genes by RNAi, she observed a striking phenotype. But to explain the significance of her findings, I have to digress for a moment and tell you about some previous work that set the stage for Alla's discovery.

This previous work goes back to 1993 when, after several years of trying, Victor Ambros' lab succeeded in cloning the lin-4 gene. ${ }^{11}$ One of the reasons lin- 4 had been so hard to clone was that the gene was tiny and did not encode a protein. Instead, the lin-4 gene appeared to encode two RNA products: an $\sim 70$ nucleotide-long RNA capable of forming a double-stranded RNA molecule with a hairpin-like structure, and a single-stranded 22 nucleotide RNA that appeared to be derived from this longer RNA (Figure 4). This short RNA was capable of binding directly to sites in the transcript of the lin-14 gene, a gene that is negatively regulated by lin- 4 during the normal course of worm development.

Even before we identified RDE-1, we were interested in the possibility of a relationship between the RNAi pathway and the lin-4 pathway. Indeed, Hiroaki had raised the concern that RNAi-defective mutants could be hard to recover as viable strains since they might also cause disruption of the lin-4 pathway. Making all of these possibilities even more exciting while we were conducting genetic screens for RNAi-deficient strains, beautiful work was published by Hamilton and Baulcombe, ${ }^{12}$ linking small RNAs of $\sim 21$ nucleotides to viral gene silencing in plants, and by Gary Ruvkun's lab, identifying a second lin-4- like worm gene, let- $7 .{ }^{13}$ Whereas lin-4 was a

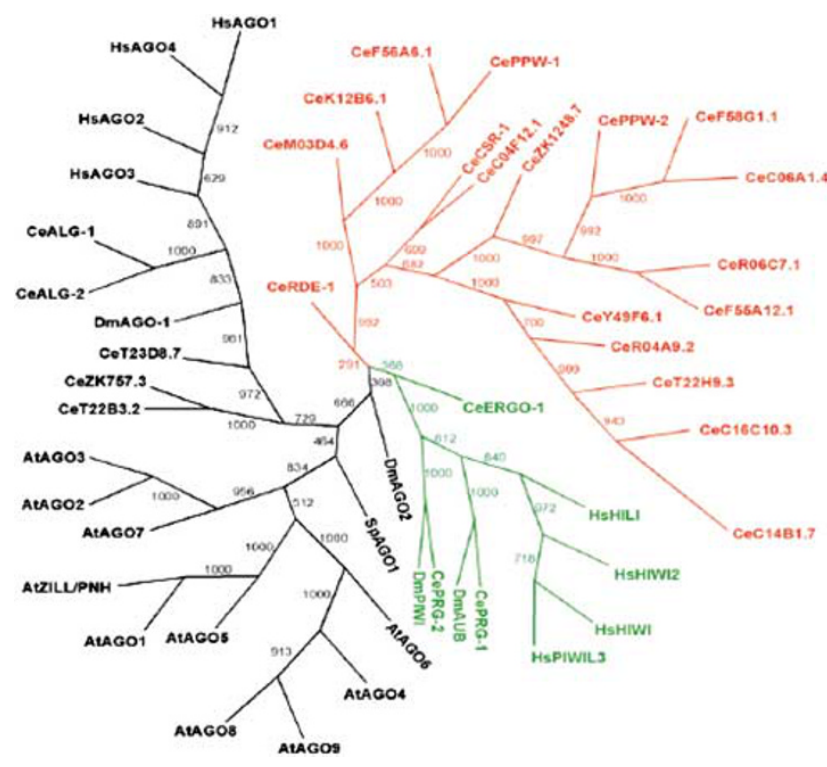

Figure 3 Phylogenetic tree of rde-1-related genes (Argonaute family).

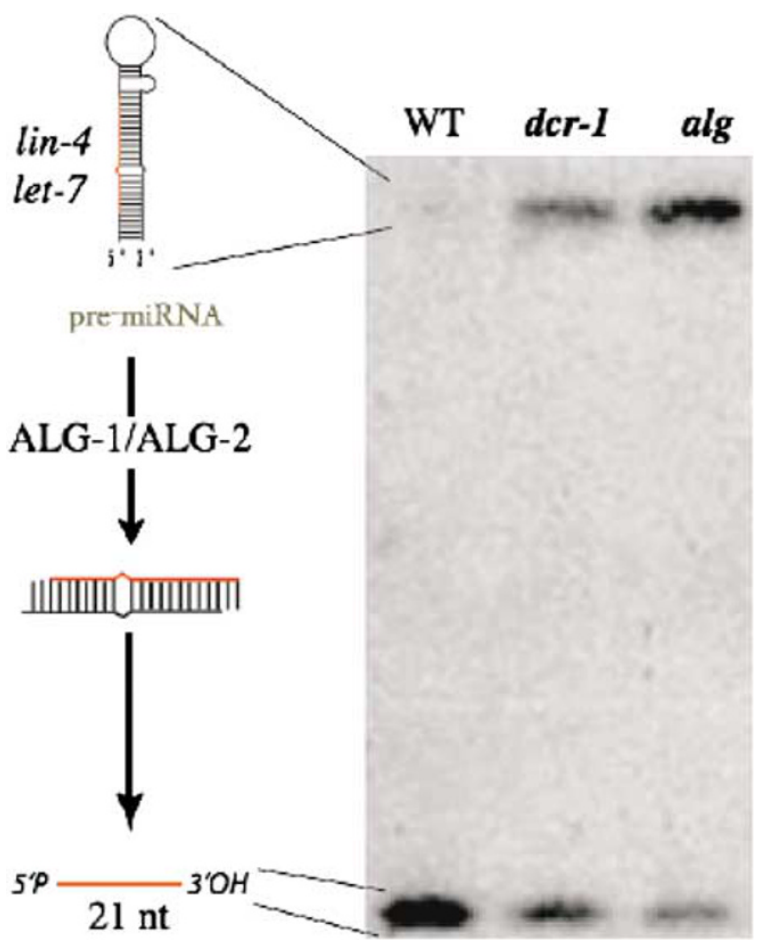

Figure 4 miRNA maturation. Gel electrophoretic separation.

worm-specific gene, it turned out that the let-7 gene had homologs in every animal, including humans. Remarkably, every single nucleotide in the 21-nucleotide mature let-7RNA products from the worm and human were identical to each other. The conservation of let-7 initiated a gold rush to find small RNA-encoding genes, now referred to as microRNAgenes, in the genomes of numerous organisms. But, despite all of the excitement, the relationship between RNA interference and microRNAs had not really been made yet. As Phil 
Sharp said at one meeting, 'It looks like a horse and smells like a horse', but there was no molecular or genetic evidence that these pathways were linked.

While this exciting work was going on in worms and plants, biochemists were making rapid progress in reconstituting elements of the silencing pathway in Drosophila cell extracts. David Bartel's group along with Phil Zamore, Tom Tuschl and Phil Sharp at MIT, and Greg Hannon's group at Cold Spring Harbor, spearheaded these efforts. ${ }^{14,15}$ They showed that activities present in Drosophila cells could process doublestranded RNA into tiny RNAs approximately 21 nucleotides long. Tom Tuschl and colleagues ${ }^{16}$ were the first to show that these small RNAs could silence gene expression in vertebrate cells. Thus, genetic studies in worms had identified small RNAs as silencing agents beginning in 1993, experimental studies of virus infections in plants identified small RNAs accumulating in infected plants, biochemical studies in fly extracts identified small RNAs in extracts, and finally experimental studies identified silencing activity in cellular assays with vertebrate cells. But were these small RNA molecules only similar in size, or did their similarity extend to mechanism? The answer to that key question was still unknown.

Alla's work provided an answer. When Alla knocked out alg-1 and alg-2, she observed a phenotype that was very similar to that observed when you knock out let-7. To confirm this connection we collaborated with Gary Ruvkun and Amy Pasquinelli, who had recently developed probes for following the processing of the lin-4 and let-7 precursor RNAs into their mature 21 nucleotide RNAs. In wild-type animals, the precursor forms are barely detectable. However, we found that, after inactivation of alg-1 and -2, this precursor accumulates to high levels while the product, the mature 21 -nucleotide RNA, is greatly diminished ${ }^{17}$ (Figure 4).

We also looked at the involvement of Dicer in this process. Dicer was identified by Greg Hannon's lab as a nuclease required for processing long double-stranded RNA into approximately 21-nucleotide fragments in Drosophila cells. We were able to show, as did several other groups, ${ }^{18,19}$ that when you knock out Dicer you also see defects in the processing of these microRNAs (Figure 4).

With these findings, the first link was established between RNA interference and a natural developmental mechanism for regulating gene expression. This was extremely exciting, and we envisioned a model (Figure 5), in which the RNAi and microRNA pathways utilized different members of the RDE-1 family and converged on Dicer. Downstream of Dicer these pathways appeared to diverge again, through the action of unknown effectors that direct different types of silencing, including mRNA destruction, transcriptional silencing and inhibition of translation. And yet, we still had not identified the RDE-1 family member involved in transposon and transgene silencing.

At that time we thought of the RDE-1 family members (also known as Argonaute proteins) as initiators of the silencing pathways. Genetic studies had placed RDE-1 at an upstream step in the pathway and, as I showed you, ALG-1 and -2 are required for processing the microRNA precursors. However, there was mounting evidence that these proteins might also function downstream in the silencing step. Definitive support for this idea came from Greg Hannon's group through a

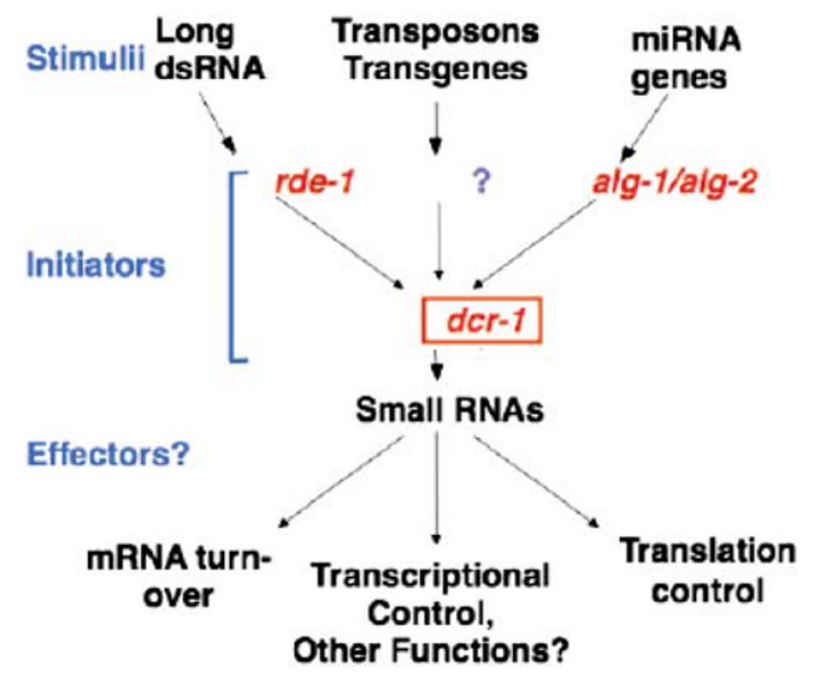

Figure 5 RNAi maturation and function.

collaboration with Ji-Joon Song and Leemor Joshua-Tor at Cold Spring Harbor. ${ }^{20}$ They showed that Argonaute proteins have structural similarity to an enzyme domain that can cut RNA, and they presented a model for how Argonaute proteins can bind the ends of the short RNAs and utilize the sequence information to find and destroy target mRNAs in the cell. These studies demonstrated that Argonaute proteins represent the long sought 'slicer' activity (or the cop) that lies at the heart of the RNA-induced silencing pathway.

We were surprised to learn that RDE-1 was probably the slicer enzyme because our genetic studies had placed RDE-1 activity at an upstream step in the pathway. However, we realized that this observation could be explained if Argonautes function more than once during RNAi in $C$. elegans. For example (Figure 6), we imagined that RDE-1 could function along with small RNAs derived from processing of the trigger dsRNA in an initial round of target mRNA cleavage. The cleaved target mRNA could then serve as a template for an RNA-dependent RNA polymerase that produces new siRNAs that could, in turn, interact with other Argonautes to mediate efficient silencing of the gene.

Experimental tests of this model were recently published. ${ }^{21}$ Surprisingly, we found that, rather than a single additional gene, multiple RDE-1 homologs function together to mediate silencing at the downstream step in the pathway. It was necessary to construct a strain containing six different Argonaute mutants to see a strong defect in RNAi. All of these functionally related genes reside within the expanded (red) family of Argonaute genes depicted in Figure 3. These downstream Argonautes are limiting for RNAi. When they are overexpressed RNAi is enhanced, and when they are inactive RNAi is decreased. These observations suggest that these genes have been amplified to mediate efficient gene silencing.

The mechanism of silencing mediated by these downstream Argonautes remains unknown. It could be through mRNA destruction, but comparison of members of this group of Argonautes to RDE-1 and other members of the family suggest that these downstream Argonautes are not likely to have an intact RNA-cleaving nuclease domain. Our studies of 


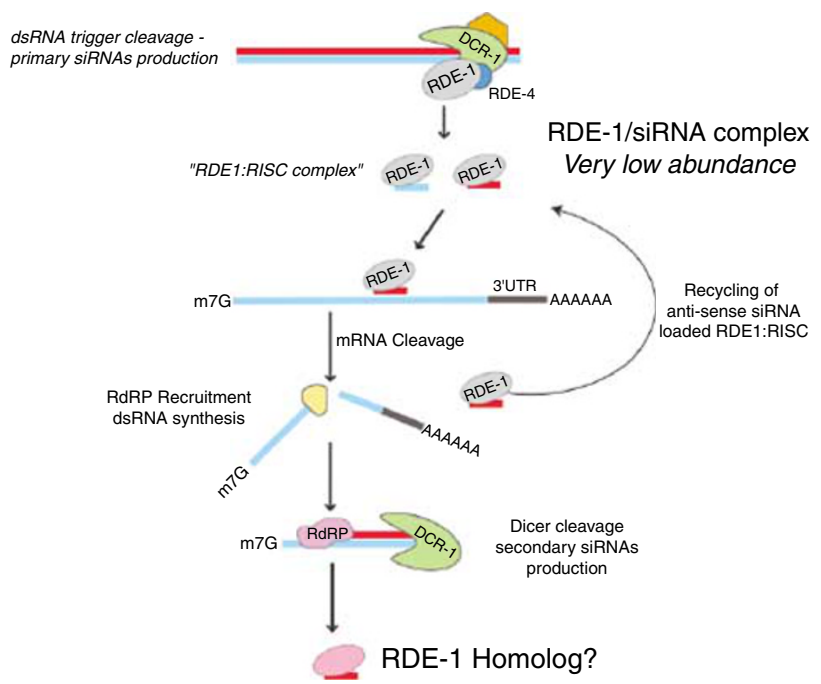

Figure 6 Function of rde-1.

these proteins indicate that they also function in endogenous silencing pathways (Figure 7), including pathways likely to have a role in silencing transposons, transgenes and other genes at the chromatin level. ${ }^{21}$

The last concept I want to discuss relates to the question of how RNAi can interact with chromatin to silence genes, and the potential importance of this mechanism for gene regulation during both development and evolution. As indicated earlier in my talk, many of the genes involved in RNAi are also required for the silencing of transgenes in the germ line. For example, the gene mut-7 was identified in our screens for genes essential for RNAi, ${ }^{6}$ but had also been identified in earlier studies as a gene required for transposon ${ }^{22}$ and transgene silencing. While RNAi appeared to have a posttranscriptional effect, several studies suggested that transgene silencing involves regulation at the level of the DNA (or more precisely, the chromatin). For example, some of the genes required for transgene silencing in $C$. elegans were related to genes of the polycomb group that interact with chromatin to direct gene silencing in other organisms. ${ }^{23}$ Beautiful direct evidence for a link between RNAi and chromatin silencing has more recently come from work in the fission yeast $S$. pombe, where a complex containing an Argonaute protein and known chromatin interacting factors has been shown to interact directly with silenced genes in the nucleus. ${ }^{24}$ To explain how RNAi could regulate DNA directly, I have to tell you a little bit about the physiological nature of DNA inside cells. Your DNA is not just lying around by itself. The unit of packaging for DNA is a protein structure called the nucleosome. The DNA wraps around the nucleosome twice, and the nucleosomes are in turn wrapped up and packaged into even thicker fibers. Chromosomes are composed of these protein/DNA fibers, also referred to as chromatin. Partly, what is achieved by packaging the DNA into chromatin is a silencing effect. Structural studies of the nucleosome core suggest that short protein tails stick out past the DNA in such a way that they are readily accessible for modification. ${ }^{25}$ The modification of these tails, and the resulting regulatory effects on gene expression, is turning out to be a fascinating subject -

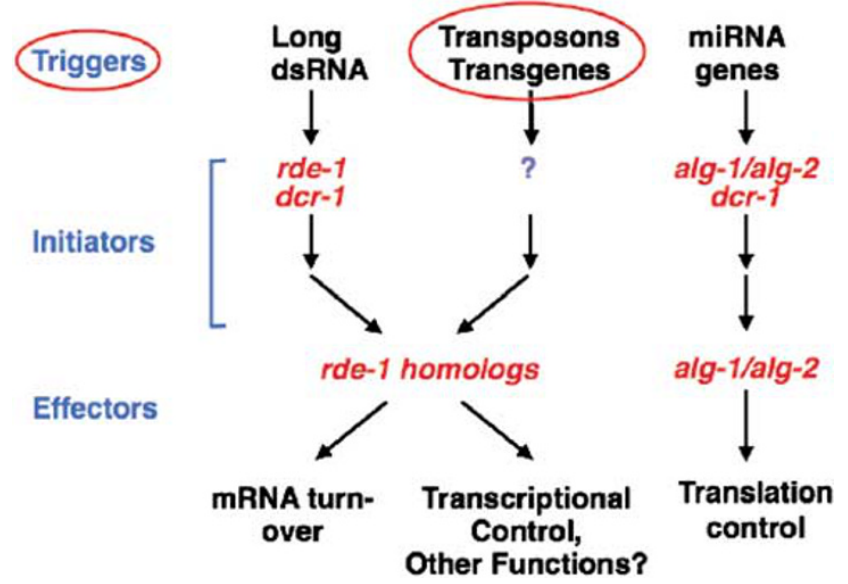

Figure 7 Silencing pathways.

one that I'm sure this committee will need to consider in the future. Interestingly, mechanisms are now emerging that explain how small RNAs can guide the modification of these chromatin tails. ${ }^{24}$ I will illustrate this mechanism with a model that could not only explain chromatin-based silencing mediated by RNAi, but also provide a mechanism for the RNA-mediated evolution concept mentioned earlier (Figure 8).

In this model the DNA (green line) is shown wrapped around the nucleosomes, which are in turn packaged into the higherorder chromatin structures. Modifications to the nucleosome tails that confer an active conformation are shown as fourpointed stars, while silencing marks are shown as multipointed red stars. In the active conformation the regulatory region of the gene, called the promoter, is free of nucleosomes and is shown bound by the RNA-polymerase complex, (the complex that produces messenger RNAs and the subject of this year's Chemistry prize). In the 'silent' region a different kind of polymerase activity is recruited. Instead of producing mRNA, this hypothetical polymerase produces transcripts that enter an RNAi-like silencing pathway. The silencing RNAs could arise by virtue of Dicer-mediated processing of doublestranded RNA. For example double-stranded RNA could form as the result of bi-directional transcription within the region, or through recruitment of an RNA-dependent RNA polymerase that recognizes some feature of the surveillance RNA. Alternatively, it is also possible that short-interfering RNAs are made directly by transcription from nucleosomal DNA and are loaded onto Argonaute proteins without going through a dsRNA intermediate. Whatever the mechanism for generating the small RNAs, the resulting Argonaute/small RNA complexes could then interact through sequence-specific interactions with nascent surveillance transcripts, or directly with the DNA, to guide chromatin-modifying enzymes back to the locus to reinforce silencing. These silencing complexes could also function in trans to silence other genes with related sequence, such as repeated members of a transposon family.

The concept of transcription occurring not simply to express the gene, but also to regulate it, is extremely powerful. Silencing marks present at low levels within 'active regions' could modulate gene expression by specifying the production 


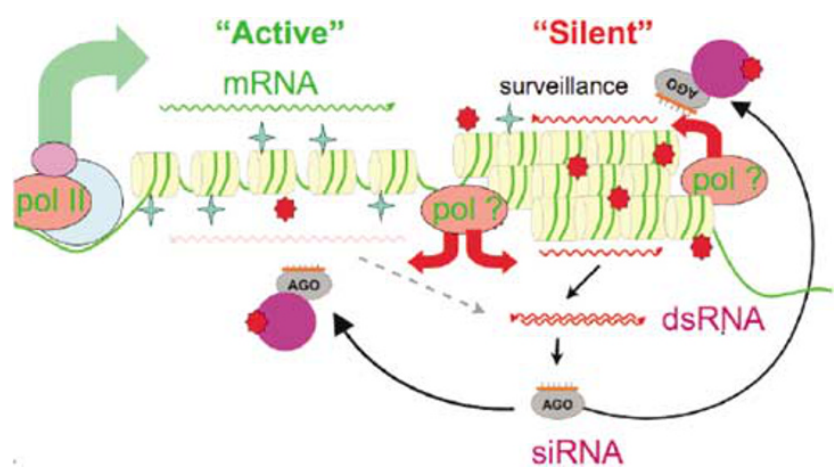

Figure 8 A chromatin-RNA feedback loop.

of intermediate levels of silencing RNAs that in turn specify an intermediate level of gene expression. According to this model, the DNA is like the hardware in a computer and the RNA/chromatin interactions are like the software. The RNA, through interactions with the chromatin, determines not only what regions of the DNA are active, but also the level of activity. When the DNA is replicated and the chromatin is disassembled, the RNA can help reinstall the silencing marks, essentially programming the resulting daughter cells to adopt gene expression patterns like the mother cell. As Weismann pointed out, asymmetric cell division could segregate this regulatory potential such that the daughter cells become different at one or many different loci. ${ }^{2}$ This mechanism could help explain how a somatic cell nucleus can be reprogrammed to undergo embryonic development after transfer into an egg. Mechanisms like this could also help explain how cells are able to maintain their gene expression programs for decades during an organism's life span.

However, and here is where evolution comes in, this RNA/ chromatin feedback mechanism could also function within the germ line. Chromatin marks in the germ line could specify a level of surveillance-RNA expression that keeps some genes off entirely, and modulates others such that, when they are activated during somatic development, their level of expression is proportional to the amount of silencing RNA produced at the locus. The feedback loop is self-sustaining but is likely to be subject to natural variations in levels. Upward or downward variations that occur naturally could be selected and transmitted in the germline from one generation to the next. This kind of reversible change in levels of gene expression could play an important role in helping organisms adjust to changes and variations in their environments.

We know from experiments in $C$. elegans that silencing induced by RNAi can be transmitted for multiple generations,$^{26}$ and that chromatin-modifying factors appear to play a role in this inheritance mechanism. ${ }^{27}$ Given the existence of these phenomena, it is tempting to speculate that all genes might continuously sample, through natural variation, different levels of heritable small RNA/chromatin interactions. Variation of this type could have a major impact on fitness and evolution, providing a rapid mechanism for evolutionary change mediated through RNA-chromatin interactions, without any underlying changes in the DNA sequence. I will end by saying we simply do not know yet how important small RNAs will turn out to be during development and evolution. I encourage you all to think about the possibilities, to learn more about biology and RNAi, and if you get inspired and excited, please join the adventure and help explore the many unknowns that are still waiting to be addressed.

1. Watson JD, Crick FH. Molecular structure of nucleic acids; a structure for deoxyribose nucleic acid. Nature 1953; 171: 737-738.

2. Weismann A 1893 The Germ-Plasm: A Theory of Heredity. New York: Charles Scribner's Sonsvxiii, 477.

3. Mayr E 1982 The Growth of Biological Thought. Cambridge, MA: The Belknap Press of Harvard University Press, 974

4. Fire A, Xu S, Montgomery MK, Kostas SA, Driver SE, Mello CC. Potent and specific genetic interference by double-stranded RNA in Caenorhabditis elegans. Nature 1998; 391: 806-811.

5. Timmons L, Fire A. Specific interference by ingested dsRNA. Nature 1998; 395: 854

6. Tabara H, Sarkissian M, Kelly WG, Fleenor J, Grishok A, Timmons L et al. The rde- 1 gene, RNA interference, and transposon silencing in C. elegans. Cell 1999; 99: 123-132.

7. Wilson JE, Connell JE, Macdonald PM. Aubergine enhances oskar translation in the Drosophila ovary. Development 1996; 122: 1631-1639.

8. Schmidt A, Palumbo G, Bozzetti MP, Tritto P, Pimpinelli S, Schafer U. Genetic and molecular characterization of sting, a gene involved in crystal formation and meiotic drive in the male germ line of Drosophila melanogaster. Genetics 1999; 151: 749-760.

9. Bohmert K, Camus I, Bellini C, Bouchez D, Caboche M, Benning C. AGO1 defines a novel locus of Arabidopsis controlling leaf development. EMBO J 1998; 17: 170-180.

10. Catalanotto C, Azzalin G, Macino G, Cogoni C. Gene silencing in worms and fungi. Nature 2000; 404: 245.

11. Lee RC, Feinbaum RL, Ambros V. The $C$ elegans heterochronic gene lin- 4 encodes small RNAs with antisense complementarity to lin-14. Cell 1993; 75: 843-854.

12. Hamilton AJ, Baulcombe DC. A species of small antisense RNA in posttranscriptional gene silencing in plants. Science 1999; 286: 950-952.

13. Reinhart BJ, Slack FJ, Basson M, Pasquinelli AE, Bettinger JC, Rougvie AE et al. The 21 nucleotide let-7 RNA regulates developmental timing in Caenorhabditis elegans. Nature 2000; 403: 901-906.

14. Zamore PD, Tuschl T, Sharp PA, Bartel DP. RNAi: double-stranded RNA directs the ATPdependent cleavage of mRNA at 21 to 23 nucleotide intervals. Cell 2000; 101: 25-33.

15. Hammond SM, Bernstein E, Beach D, Hannon GJ. An RNA-directed nuclease mediates post-transcriptional gene silencing in Drosophila cells. Nature 2000; 404: 293-296.

16. Elbashir SM, Harborth J, Lendeckel W, Yalcin A, Weber K, Tuschl T. Duplexes of 21-nucleotide RNAs mediate RNA interference in cultured mammalian cells. Nature 2001; 411: 494-498.

17. Grishok A, Pasquinelli AE, Conte D, Li N, Parrish S, Ha I et al. Genes and mechanisms related to RNA interference regulate expression of the small temporal RNAs that control $C$. elegans developmental timing. Cell 2001; 106: 23-34.

18. Hutvagner G, McLachlan J, Pasquinelli AE, Balint E, Tuschl T, Zamore PD. A cellular function for the RNA-interference enzyme Dicer in the maturation of the let-7 small temporal RNA. Science 2001; 293: 834-838.

19. Ketting RF, Fischer SE, Bernstein E, Sijen T, Hannon GJ, Plasterk RH. Dicer functions in RNA interference and in synthesis of small RNA involved in developmental timing in C. elegans. Genes Dev 2001; 15: 2654-2659.

20. Song JJ, Smith SK, Hannon GJ, Joshua-Tor L. Crystal structure of Argonaute and its implications for RISC slicer activity. Science 2004; 305: 1434-1437.

21. Yigit E, Batista PJ, Bei Y, Pang KM, Chen CC, Tolia NH et al. Analysis of the C. elegans Argonaute family reveals that distinct Argonautes act sequentially during RNAi. Cell 2006; 127: $747-757$.

22. Ketting RF, Haverkamp TH, van Luenen HG, Plasterk RH. MUT-7 of $C$. elegans, required for transposon silencing and RNA interference, is a homolog of Werner syndrome helicase and RNaseD. Cell 1999; 99: 133-141.

23. Kelly WG, Fire A. Chromatin silencing and the maintenance of a functional germline in Caenorhabditis elegans. Development 1998; 125: 2451-2456.

24. Verdel A, Jia S, Gerber S, Sugiyama T, Gygi S, Grewal SI et al. RNAi mediated targeting of heterochromatin by the RITS complex. Science 2004; 303: 672-676.

25. Richmond TJ, Davey CA. The structure of DNA in the nucleosome core. Nature 2003; 423: $145-150$.

26. Grishok A, Tabara H, Mello CC. Genetic requirements for inheritance of RNAi in C. elegans. Science 2000; 287: 2494-2497.

27. Vastenhouw NL, Brunschwig K, Okihara KL, Muller F, Tijsterman M, Plasterk RH. Gene expression: long-term. 